\title{
Aliesterase no sôro de alguns animais de laboratório
}

\author{
por \\ Maria Isabel Mello (*)
}

Os têrmos "lipase" e "esterase" são empregados indistintamente na lite ratura, ainda que estas enzimas sejam entidades separadas.

Em 1932, Cherry e Crandall propuseram o têrmo "lipase" para a enzima (ou enzimas) capaz de desdobrar as gorduras verdadeiras e os óleos, e o têrmo "esterase" para a enzima (ou enzimas) que agem sôbre ésteres simples. O emprêgo de substratos sintéticos e inhibidores específicos trouxe um grande impulso na caracterização destas enzimas (1), (2), (3), (4), (5).

Recentemente, Adams e Whittaker conseguiram demonstrar que as enzimas que catalizam a hidrólise dos ésteres alifáticos tais como o butirato de metila e a tributirina, são "esterases alifáticas" ou "aliesterases". Di ferem das "lipases" que ainda que possam hidrolizar triglicerides mais simples hidrolizam ácidos graxos de cadeias longas (6).

A "esterase", "aliesterase" é um constituinte normal do sôro, enquanto que a "lipase" não o é (Richter e Croft) (7).

Têm sido propostos para a determinação da lipase métodos biológicos, físicos e químicos (8), (9), (10).

Em 1947, Huggins e Lapides publicaram um novo método químico para a determinação da "esterase" do sôro, "aliesterase". A enzima tamponada em pH ótimo, age sôbre o substrato, que é no caso um éster incolôr de para nitrofenol. A ação enzimática é determinada colorimetricamente baseada no teôr de para-nitrofenol libertado (11). No presente trabalho foi usado como substrato o propionato de para-nitrofenol.

É difícil encontrar na literatura valores normais para a "esterase" do sôro de animais de laboratório. Huggins e Moulton investigaram o modo de distribuição da esterase nos vários tecidos e no sangue do rato (12).

Procuramos então determinar os válores normais não só para o sôro do rato, como do hamster, cobaia e coelho.

(*) Laboratório de Bioquimica, Instituto Oswaldn Cruz. 


\section{MÉTODOS}

Tôdas as amostras foram obtidas por punção cardíaca. A determinação da aliesterase foi feita pelo método de Huggins e Lapides já descrita por nós em trabalho anterior ligeiramente modificado (13). O substrato usado foi o propionato de para-nitrofenol preparado pela técnica de Spasov (14) (§), e o sôro tamponado em $\mathrm{pH}$ 7.0. As leituras foram feitas no espectrofotômetro de Beckan e no comprimento de onda de $400 \mathrm{~m}_{\mu}$ Tôdas as determinações foram feitas em $0.1 \mathrm{ml}$ de sôro e em duplicata.

\section{RESULTADOS}

Foram encontrados dois padrões diferentes para a atividade esterásica do sôro do rato de acôrdo com o sexo.

É interessante notar que os valores obtidos com sôro de ratas fêmeas normais apresentam flutuações ligadas ao ciclo estral.

A média global foi de $11.6 \pm 4.01$ com um $\mathrm{E}=0.83$. O estudo em separado dos vários grupos evidencia a influência da fase estral sôbre o teôr da esterase no sôro. Os valores mais altos foram obtidos de ratas em proestro enquanto os animais em diestro apresentaram os menores valores. Estas variações poderão ser melhor observadas nos quadros I e II.

QUADRO II

ALIESTERASEMIA E FASE ESTRAL

\begin{tabular}{|c|c|c|c|c|}
\hline FASES DO CICLO & $\begin{array}{l}\text { N. }{ }^{\circ} \text { DE } \\
\text { ANIMAIS }\end{array}$ & M & $\sigma$ & $\mathrm{E}_{\mathrm{M}}$ \\
\hline $\begin{array}{l}\text { Diestro.......... } \\
\text { Proestro........ } \\
\text { Estro.......... } \\
\text { Metaestro..... }\end{array}$ & $\begin{array}{l}8 \\
7 \\
4 \\
4\end{array}$ & $\begin{array}{l}7.35 \\
15.54 \\
14.75 \\
10.075\end{array}$ & $\begin{array}{l}1.82 \\
2.25 \\
1.25 \\
1.00\end{array}$ & $\begin{array}{l}0.64 \\
0.86 \\
0.65 \\
0.50\end{array}$ \\
\hline TOTAL. & 23 & 11.6 & 4.01 & 0.83 \\
\hline
\end{tabular}

Com se pode observar há uma diferença acentuada nos valores para a esterasemia nas várias fases do ciclo. Isto demonstra que a concentração da esterase no sôro encontra-se intimamente ligada à produção ou metabolismo

( ${ }^{\star}$ Agradecemos ao Dr. Augusto Cid de Mello Perissé a preparação deste substrato. 
QUADRO I

ALIESTERASE NO SORO DE RATOS NORMAIS (FEMEAS)

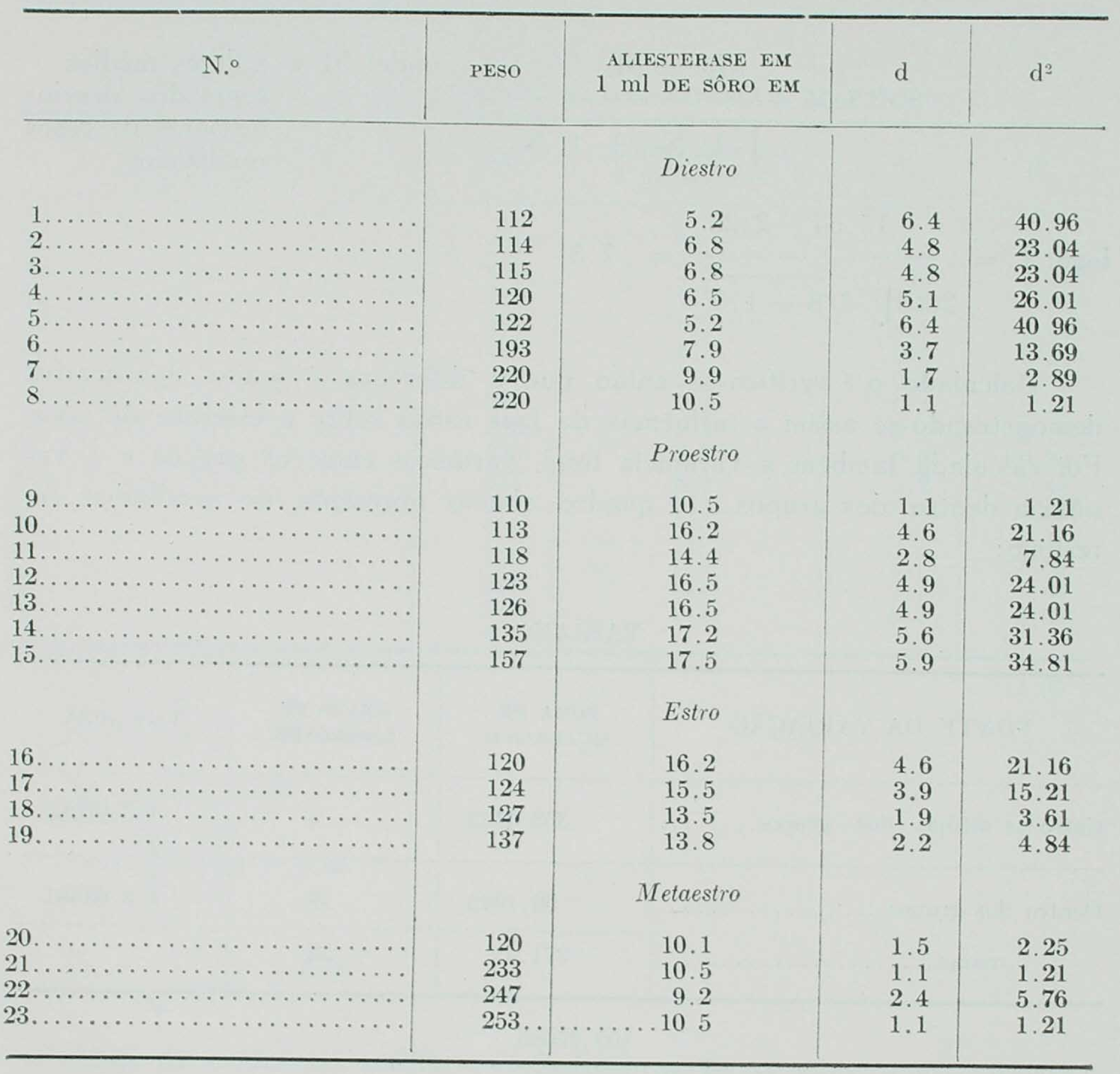

$\mathrm{M}=\frac{(\mathrm{x})}{\mathrm{N}}$

371.45

$\mathrm{M}=11.6 \pm 4.01$

$\sigma=\sqrt{\frac{(\mathrm{d})^{2}}{\mathrm{~N}}}=4.01$

${ }^{E_{\mathbf{M}}} \| \frac{\sigma}{\sqrt{\mathrm{N}}}=0.83$ 
dos estrogênios. Uma análise dêstes valores pela aplicação do test da significância demonstrará melhor essas váriaçōes:

$$
t=\frac{M_{1}-M_{2}}{\sqrt{1 / N_{1} \pm 1 / N_{2}}}
$$$$
\text { logo, } t=\frac{15,54-2,25}{2.19 \sqrt{1 / 8+1 / 7}}=7.3
$$

onde: $M=$ valores médios

$\mathrm{t}=$ soma dos desvios

$\mathrm{N}=$ números dé $\operatorname{casos}$ analizados,

Calculado o $t$ verifica-se então que a diferença é muito significativa demonstrando-se assim a influência da fase estral sôbre a esterase do sôro. Foi calculada também a variância total, variância entre os grupos e a variância dentro dos grupos. O quadro abaixo apresenta os resultados em resumo:

\section{VARIANCIA}

\begin{tabular}{|c|c|c|c|}
\hline FONTE DA VARIAÇÃO & $\begin{array}{c}\text { SOMA DE } \\
\text { QUADRADOS }\end{array}$ & $\begin{array}{l}\text { GRAUS DE } \\
\text { LIBERDADE }\end{array}$ & VARIANCIA \\
\hline Entre as médias dos grupos.. & 302.3125 & 3 & 100,77083 \\
\hline \multirow{2}{*}{$\begin{array}{r}\text { Dentro dos grupos. } \\
\text { тотаL. } \ldots \ldots \ldots \ldots \ldots \ldots \ldots \ldots\end{array}$} & 69,1375 & 19 & 3. 63881 \\
\hline & 371.45 & 22 & - \\
\hline
\end{tabular}

$$
\mathrm{F}=\frac{100,77083}{3,638816}=27,6
$$

\section{IN'TERPRETAÇÃO}

Para $V_{1}=3$ e $V_{2}=19$, as tábuas de Snedecor dão um valôr de $\mathrm{F}$ entre 3,10 e 3,16 no ponto de significância de $5 \%$. O valôr observado, fi cando muito acima, é altamente significativo.

Tendo as ratas sido classificadas nos quatro grupos segundo o critério da fase do ciclo estral, conclue-se que as divergências entre as diversas médias da esterasemia estão ligadas ao metabolismo estrogênico. 
Os ratos machos normais apresentam valores mais baixos e uniformes como se pode vêr no quadro III.

QUADRO III

ALIESTERASE NO SORO DE RATOS NORMAIS MACHOS

\begin{tabular}{|c|c|c|}
\hline $\mathrm{N}^{\circ}$ & PESO & $\begin{array}{l}\text { ALIESTERASE EM } \\
1 \mathrm{ml} \text { DE SÔRO }\end{array}$ \\
\hline 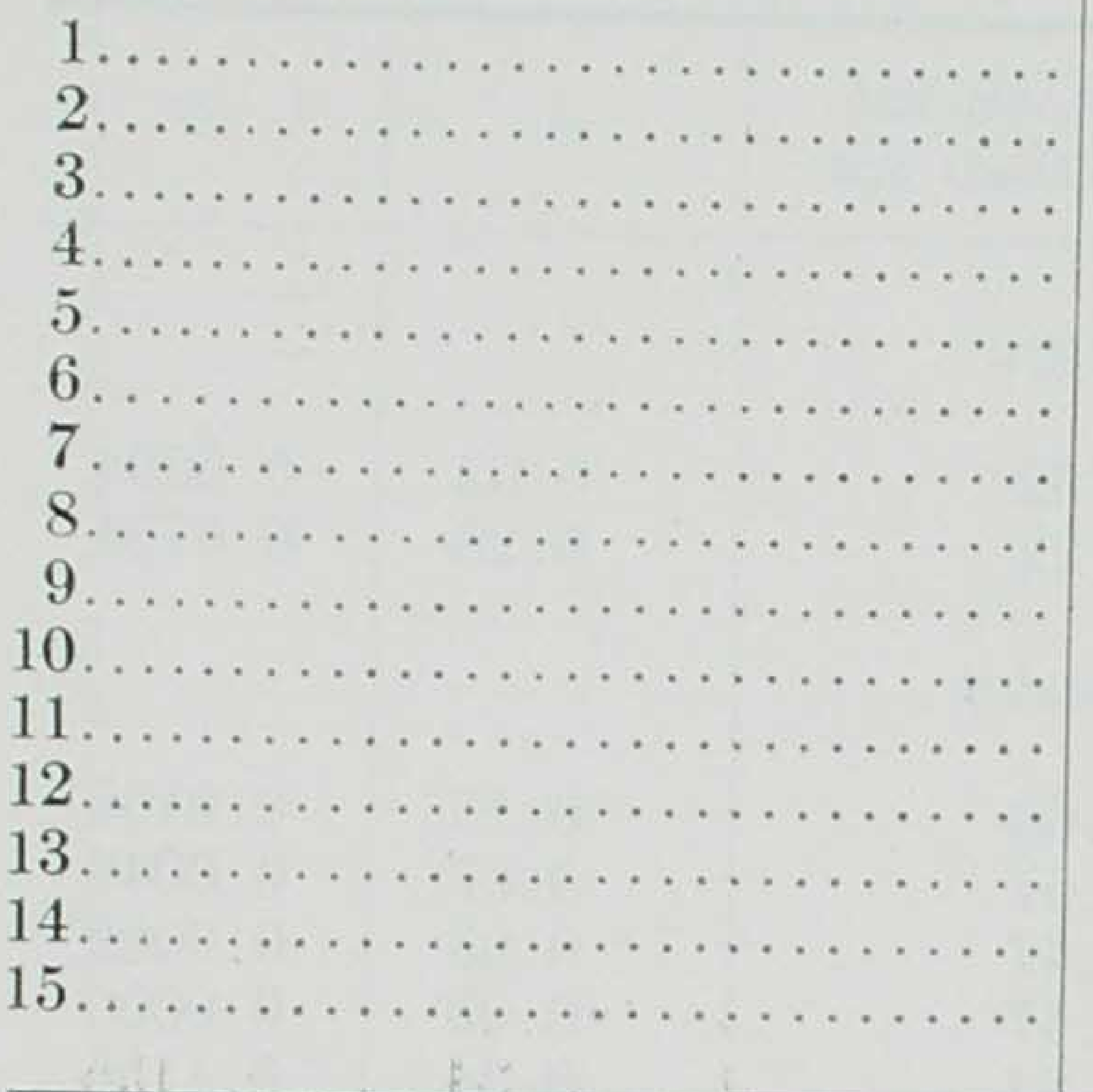 & $\begin{array}{l}220 \\
230 \\
250 \\
220 \\
210 \\
210 \\
214 \\
220 \\
220 \\
227 \\
23 ? \\
234 \\
250 \\
273 \\
280\end{array}$ & $\begin{array}{l}7.1 \\
7.9 \\
9.1 \\
6.7 \\
6.3 \\
7.3 \\
7.3 \\
7.3 \\
6.3 \\
8.1 \\
6.3 \\
7.3 \\
5.2 \\
5.2 \\
6.7\end{array}$ \\
\hline
\end{tabular}

$$
\begin{aligned}
& \mathrm{M}=\frac{(\mathrm{x})}{\mathrm{N}} \\
& \mathrm{M}=6.9 \pm 0.99 \\
& \sigma=\sqrt{\frac{(\mathrm{d})^{2}}{\mathrm{~N}}}=0.99
\end{aligned}
$$

$$
\mathrm{E}_{\mathrm{M}}=\frac{\sigma}{\sqrt{\mathrm{N}}}=0.26
$$

Afim de evidenciar melhor a influência do sexo sôbre a esterasemia, calculamos também o $t$ con a média global obtica para as fêmeas tabeladas no quadro I e a média dos valores obtidos para os machos do quadro III:

$$
t=\frac{M_{1}-M_{2}}{\frac{1 / N_{1} \pm 1 / N_{2}}{3,27} \overline{/ 1 / 15+1 / 23}}=0.4 .4
$$

Donde se deduz que o valôr de $t$ comparando os grupos macho e fêmea também é significativo.

$\mathrm{Na}$ cobaia notam-se diferençäs nas médias. Os valores para as fêmeas foram mais ou menos constantes. Deve-se assinalar entretanto serem as 
fêmeas usadas para estas dosagens, de apenas dois meses de idade e de procedências diversas. Os machos eram animais de 3 e 4 meses e todos pertencentes à mesma colônia.

Nos quadros IV e $\mathrm{V}$ encontram-se tabelados os valores obtidos.

QUADRO IV

ALIESTERASE NO SORO DE COBAIAS NORMAIS (FEMEAS)

\begin{tabular}{|c|c|c|c|c|}
\hline $\mathrm{N} .{ }^{\circ}$ & PESO & $\begin{array}{l}\text { ALIESTERASE EM } \\
1 \mathrm{ml} \text { DE SÔRO EM }\end{array}$ & d & $d^{2}$ \\
\hline $\begin{array}{l}1 \\
2 \ldots \ldots \ldots \ldots \ldots \ldots \ldots \ldots \ldots \ldots \ldots \ldots \ldots\end{array}$ & $\begin{array}{l}230 \\
294\end{array}$ & $\begin{array}{c}\text { Diestro } \\
\\
4.52 \\
4.22 \\
\text { Proestro }\end{array}$ & $\begin{array}{l}0.02 \\
0.28\end{array}$ & $\begin{array}{l}0.0004 \\
0.0784\end{array}$ \\
\hline 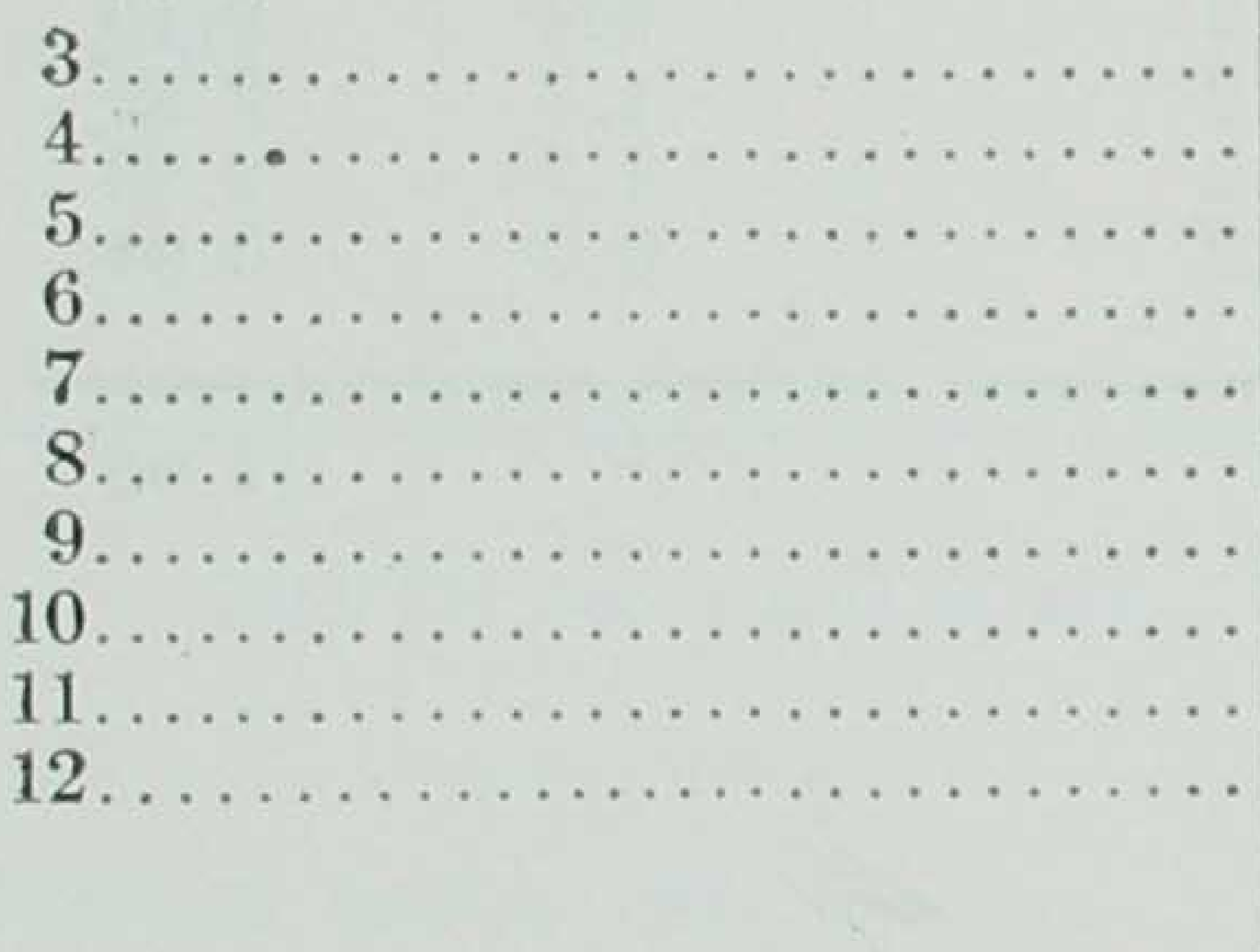 & $\begin{array}{l}190 \\
199 \\
204 \\
206 \\
209 \\
211 \\
220 \\
227 \\
278 \\
178\end{array}$ & $\begin{array}{l}4.63 \\
4.57 \\
4.47 \\
4.47 \\
4.84 \\
4.52 \\
4.63 \\
4.30 \\
4.57 \\
4.73 \\
\text { Estro }\end{array}$ & $\begin{array}{l}0.13 \\
0.07 \\
0.03 \\
0.03 \\
0.34 \\
0.02 \\
0.13 \\
0.20 \\
0.07 \\
0.23\end{array}$ & $\begin{array}{l}0.0169 \\
0.0049 \\
0.0009 \\
0.0009 \\
0.1156 \\
0.0004 \\
0.0169 \\
0.0400 \\
0.0049 \\
0.0529\end{array}$ \\
\hline 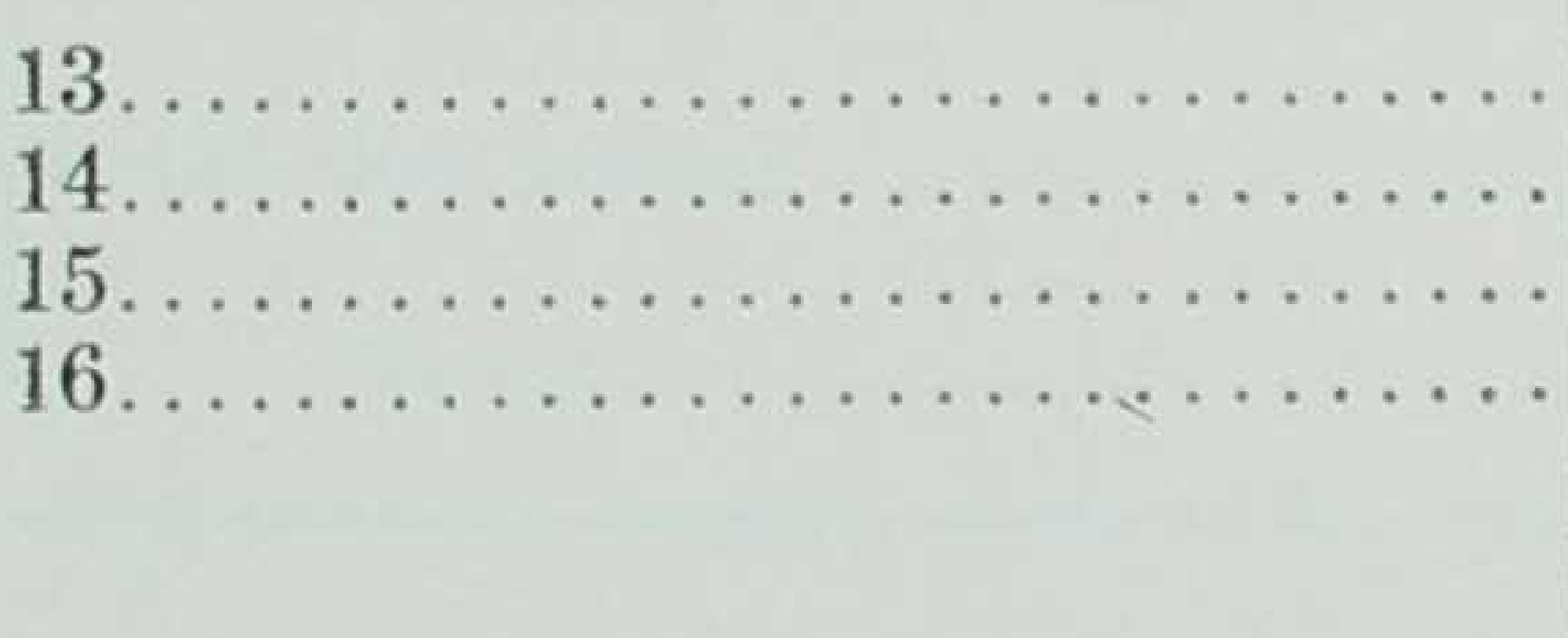 & $\begin{array}{l}245 \\
247 \\
257 \\
280\end{array}$ & $\begin{array}{c}4.42 \\
4.52 \\
4.30 \\
4.42 \\
\text { Metaestro }\end{array}$ & $\begin{array}{l}0.08 \\
0.02 \\
0.20 \\
0.08\end{array}$ & $\begin{array}{l}0.0064 \\
0.0004 \\
0.0400 \\
0.0064\end{array}$ \\
\hline $17 \ldots \ldots \ldots \ldots \ldots \ldots$ & 292 & 4.52 & 0.02 & 0.0004 \\
\hline
\end{tabular}

$$
\begin{aligned}
& \mathrm{M}_{\mathrm{L}}=\frac{(\mathrm{x})}{\mathrm{N}} \\
& \mathrm{M}_{\mathrm{l}}=4.50 \pm 0.15 \\
& \sigma_{1}=\sqrt{\frac{(\mathrm{d})^{2}}{\mathrm{~N}}}=0.15 \\
& \mathrm{E}_{\mathrm{M}_{1}}=\frac{\sigma}{\sqrt{\mathrm{N}}}=0036
\end{aligned}
$$


QUADRO V

ALIESTERASE NO SORO DE COBAIAS NORMAIS (MACHOS)

\begin{tabular}{|c|c|c|c|c|}
\hline $\mathrm{N} .{ }^{\circ}$ & PESO & $\begin{array}{l}\text { ALIESTERASE EM } \\
1 \mathrm{ml} \text { DE SORO }\end{array}$ & $\mathrm{d}$ & $d^{2}$ \\
\hline 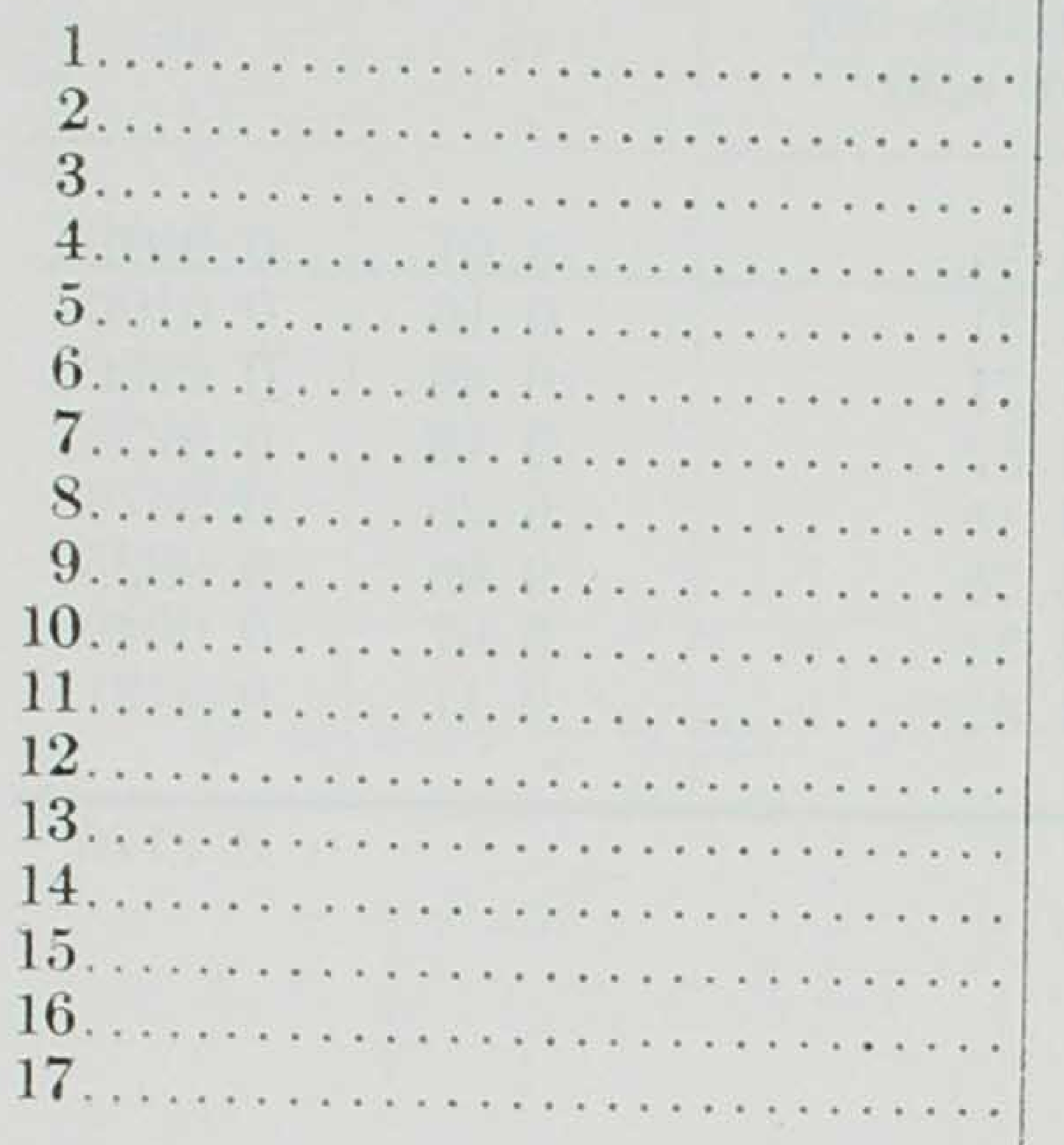 & $\begin{array}{l}377 \\
410 \\
410 \\
417 \\
444 \\
450 \\
468 \\
500 \\
185 \\
248 \\
268 \\
295 \\
300 \\
312 \\
315 \\
316 \\
376\end{array}$ & $\begin{array}{l}3.94 \\
3.97 \\
4.00 \\
3.94 \\
4.42 \\
3.84 \\
4.31 \\
3.90 \\
4.21 \\
4.34 \\
4.31 \\
4.57 \\
4.52 \\
4.73 \\
4.47 \\
4.42 \\
4.90\end{array}$ & $\begin{array}{l}0.34 \\
0.31 \\
0.28 \\
0.34 \\
0.14 \\
0.44 \\
0.03 \\
0.38 \\
0.07 \\
0.06 \\
0.03 \\
0.29 \\
0.24 \\
0.45 \\
0.19 \\
0.14 \\
0.62\end{array}$ & $\begin{array}{l}0.1156 \\
0.0561 \\
0.0784 \\
0.1156 \\
0.0196 \\
0.1936 \\
0.0009 \\
0.1444 \\
0.0049 \\
0.0036 \\
0.0009 \\
0.0841 \\
0.0576 \\
0.2025 \\
0.0361 \\
0.0196 \\
0.3844\end{array}$ \\
\hline
\end{tabular}

$$
\begin{aligned}
\mathrm{M}_{2} & =\frac{(\mathrm{x})}{\mathrm{N}} \\
\mathrm{M}_{2} & =4.28 \pm 0.30 \\
\sigma_{2} & =\sqrt{\frac{(\mathrm{d})^{2}}{\mathrm{~N}}}=0.30 \\
\mathrm{E}_{\mathrm{M}_{2}} & =\frac{\sigma}{\sqrt{\mathrm{N}}}=0.073 \\
\mathrm{t} & =-\frac{M_{1}-\mathrm{M}_{2}}{\sqrt{1 / \mathrm{N}_{1} \pm 1 / \mathrm{N}_{2}}}=\frac{4,50-4,23}{0,24 \times 0,34}=2.6
\end{aligned}
$$

Apesar dos valores apresentados pelas fêmeas serem pouco mais elevados, calculando-se o $t$ verifica-se ser esta diferença significante. 
Para os hamsters e coelhos, foram calculados apenas as médias e desvios. Entretanto, nos quadros VI, VII e. VIII podem ser apreciados os valores registrados para estes animais.

QUADRO VI

ALIESTERASE NO SORO DE COELHOS NORMAIS (MACHOS)

\begin{tabular}{|c|c|c|c|c|c|}
\hline$i^{1}+\pi$ & No & PESO & $\begin{array}{l}\text { ALIESTERASE EM } \\
1 \mathrm{ml} \text { DE SÔRO }\end{array}$ & d & $d^{2}$ \\
\hline $\begin{array}{l}1 \ldots \ldots \\
2 \ldots \\
3 \ldots \\
4 \\
5 \ldots \\
6 \ldots \\
7 \ldots \ldots \\
8 \ldots\end{array}$ & 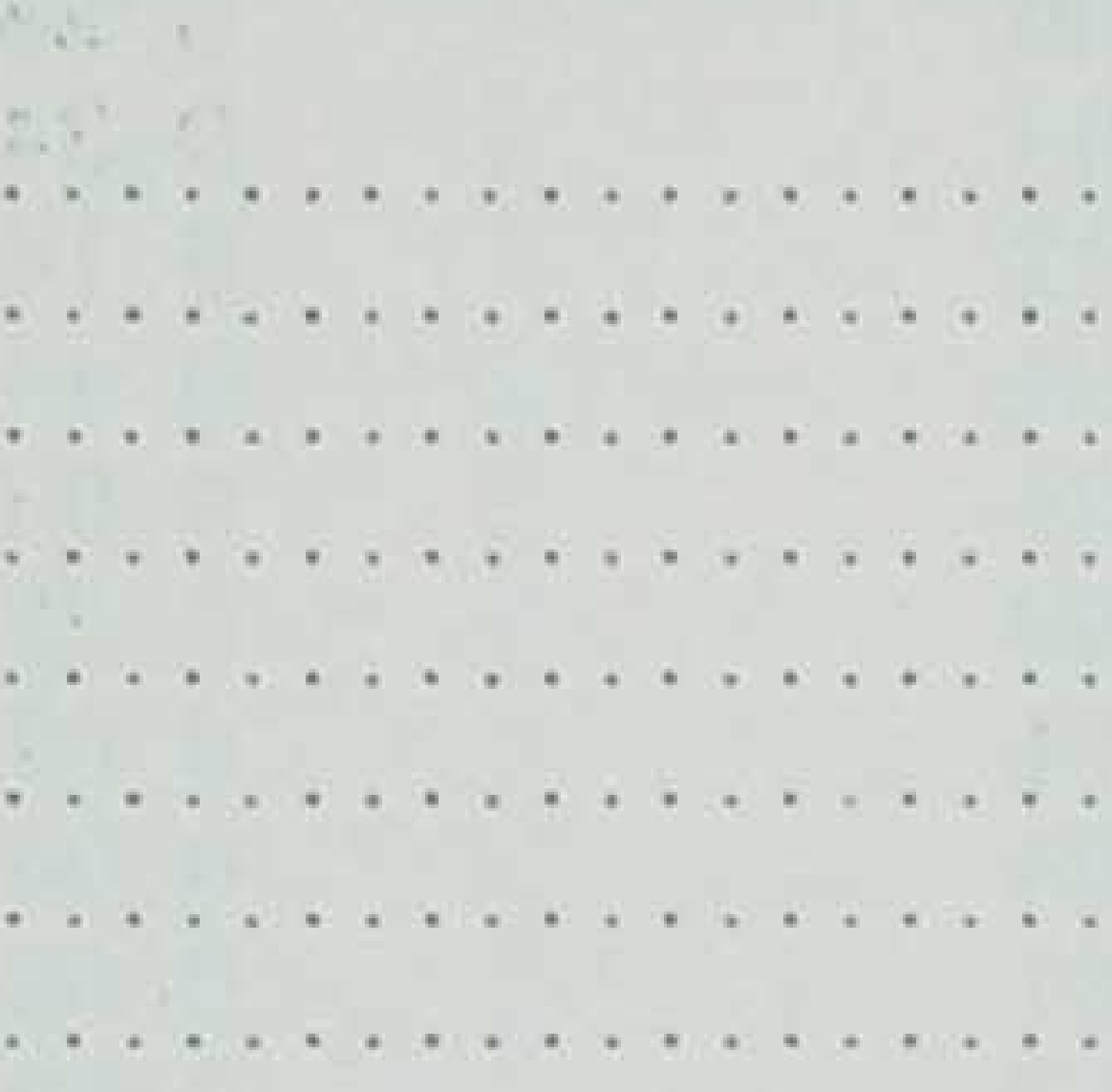 & $\begin{array}{l}1.800 \\
1.800 \\
1.880 \\
2.000 \\
2.120 \\
2.230 \\
2.420 \\
3.200\end{array}$ & $\begin{array}{l}4.34 \\
4.31 \\
4.71 \\
4.57 \\
4.15 \\
4.36 \\
4.34 \\
4.52\end{array}$ & $\begin{array}{l}0.07 \\
0.10 \\
0.30 \\
0.16 \\
0.26 \\
0.05 \\
0.07 \\
0.11\end{array}$ & $\begin{array}{l}0.0049 \\
0.0100 \\
0.0900 \\
0.0256 \\
0.0676 \\
0.0025 \\
0.0049 \\
0.0121\end{array}$ \\
\hline
\end{tabular}

$$
\begin{aligned}
\mathrm{M} & =\frac{(\mathrm{x})}{\mathrm{N}} \\
\mathrm{M} & =4.41 \pm 0.16 \\
\sigma & =\sqrt{\frac{(\mathrm{d})^{2}}{\mathrm{~N}}}=0.16 \\
\mathrm{E}_{\mathrm{M}} & =\frac{\sigma}{\sqrt{\mathrm{N}}}=0.058
\end{aligned}
$$

\begin{tabular}{|c|c|c|c|c|}
\hline $\mathrm{N} .{ }^{\circ}$ & PESO & $\begin{array}{l}\text { ALIESTERASE EM } \\
1 \mathrm{ml} \text { DE SÔRO }\end{array}$ & d & $d^{2}$ \\
\hline 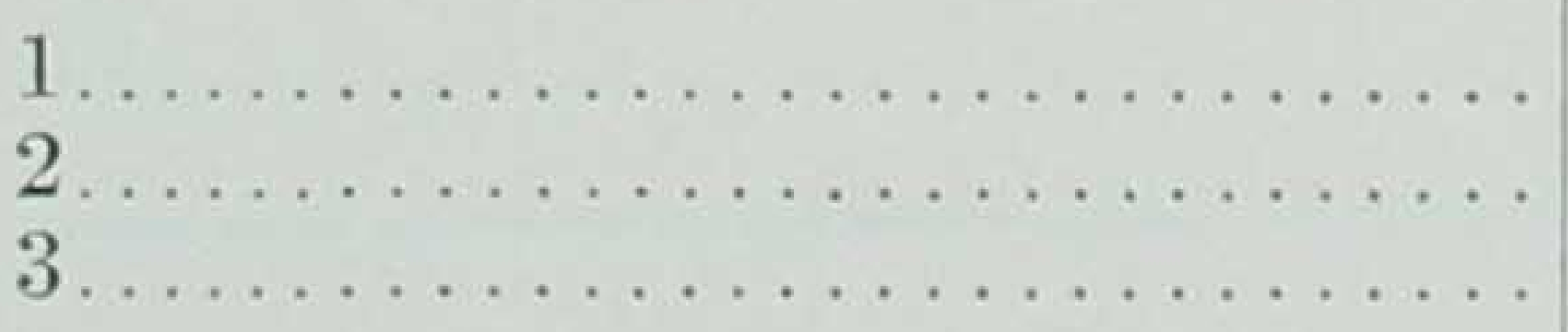 & $\begin{array}{l}102 \\
103 \\
103\end{array}$ & $\begin{array}{l}4.10 \\
4.21 \\
4.00\end{array}$ & $\begin{array}{l}0.0 \\
0.11 \\
0.10\end{array}$ & $\begin{array}{l}0.0121 \\
0.0100\end{array}$ \\
\hline
\end{tabular}

QUADRO VII

ALIESTERASE NO SORO DE HAMSTERS NORMAIS (MACHOS)

$$
\begin{aligned}
\mathrm{M} & =\frac{(\mathrm{x})}{\mathrm{N}} \\
\mathrm{M} & =4.10 \pm 0.085 \\
\sigma & =\sqrt{\frac{(\mathrm{d})^{2}}{\mathrm{~N}}}=0.085 \\
\mathrm{E}_{\mathrm{M}} & =\frac{{ }^{\sigma}}{\sqrt{\mathrm{N}}}=0.049
\end{aligned}
$$


QUADRO VIII

ALIESTERASE NO SORO DE HAMSTERS NORMAIS (FEMEAS)

\begin{tabular}{|c|c|c|c|c|}
\hline N. ${ }^{\circ}$ & PESO & $\begin{array}{l}\text { ALIESTERASE ENI } \\
1 \mathrm{ml} \text { DE SÔRO }\end{array}$ & d & $d^{2}$ \\
\hline 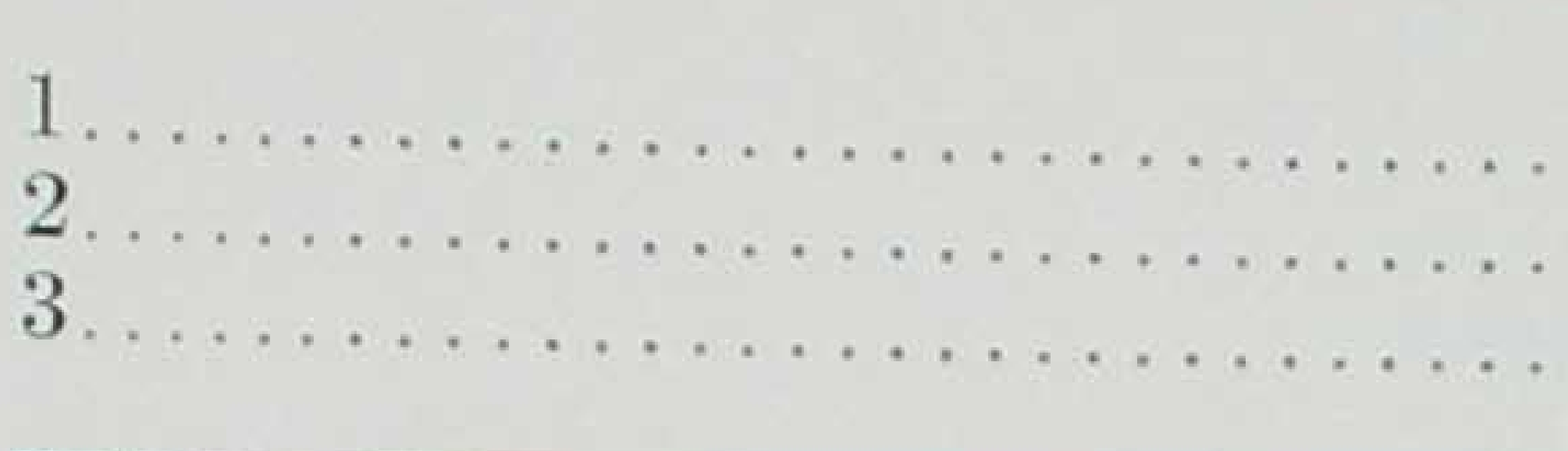 & $\begin{array}{r}110 \\
76 \\
65\end{array}$ & $\begin{array}{l}3.94 \\
3.49 \\
4.00\end{array}$ & $\begin{array}{l}0.13 \\
0.32 \\
0.19\end{array}$ & $\begin{array}{l}0.0169 \\
0.1024 \\
0.0361\end{array}$ \\
\hline
\end{tabular}

$$
\begin{aligned}
\mathrm{M} & =\frac{(\mathrm{x})}{\mathrm{N}} \\
\mathrm{M} & =3.81 \pm 0.227 \\
\sigma & =\sqrt{\frac{(\mathrm{d})^{2}}{\mathrm{~N}}}=0.227 \\
\mathrm{E}_{\mathrm{M}} & =\frac{\sigma}{\sqrt{\mathrm{N}}}=0.133
\end{aligned}
$$

\section{SLIMÁRIO}

A esterase, aliesterase, foi determinada no sôro de 38 ratos de ambos os sexos, 6 hamsters, 34 cobaias e 8 coelhos, pela técnica de Huggins e Lapides. Os resultados obtidos para os machos e fêmeas foram tabelados em sepa rado. As fêmeas apresentaram uma esterasemia maior que os machos, com uma média global de $11.6 \mathrm{U} / \mathrm{ml} \pm 4.01$ e um erro padrão de 0.83 en quanto que para os machos a média foi de $6.9 \mathrm{U} / \mathrm{ml} \pm 0.99 \mathrm{com}$ um erro padrão de 0.26. A significância calculada pelo $t$ foi de 4.4 Isto demonstra a interferência da aliesterase na produção ou no metabolismo dos estrogênios.

Os valores médios encontrados para as cobaias foram $4.50 \mathrm{U} / \mathrm{ml} \pm$ 0.15 , com um êrro padrão de 0.036 para as fêmeas e $4.28 \mathrm{U} / \mathrm{ml} \pm 0.30$ com um êrro padrão de 0.073 para os machos, sendo a significância (t) de 2.6.

Para os coelhos (machos) a média foi de $4.41 \mathrm{U} / \mathrm{ml} \pm 0.16$, com um êrro padrão de 0.058 enquanto para os hamsters os valores foram de 4.01 
$\mathrm{U} / 1 \pm 0.085$, êrro padrão de 0.049 para os machos e $3.81 \mathrm{U} / \mathrm{ml} \pm 0.227$, êrro padrão de 0.133 para as fêmeas.

Os animais castrados mostram uma diminuição progressiva da esterase no sôro, enquanto que nos castrados e tratados com estrogênios, êstes valores atingem o teôr normal. Os resultados obtidos nestes casos serão objeto de publicação ulterior.

Agradecemos ao Dr. Washington de Almeida a análise estatística do presente trabalho. 